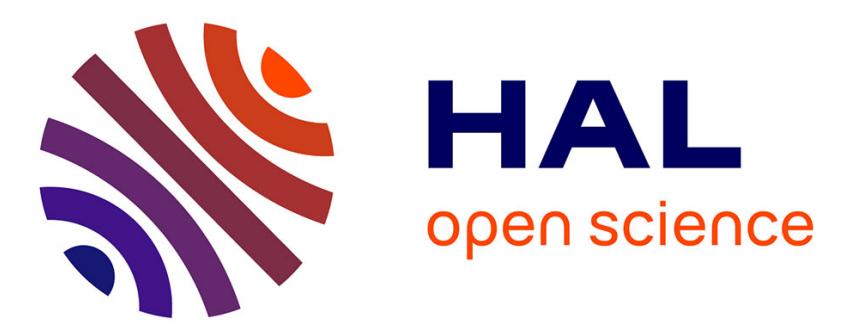

\title{
Implication of epigenetics in pancreas development and disease
}

Evans Quilichini, Cécile Haumaitre

\section{To cite this version:}

Evans Quilichini, Cécile Haumaitre. Implication of epigenetics in pancreas development and disease. Best Practice and Research: Clinical Endocrinology and Metabolism, 2015, 29 (6), pp.883-898. 10.1016/j.beem.2015.10.010 . hal-01227978

\section{HAL Id: hal-01227978 https://hal.sorbonne-universite.fr/hal-01227978}

Submitted on 12 Nov 2015

HAL is a multi-disciplinary open access archive for the deposit and dissemination of scientific research documents, whether they are published or not. The documents may come from teaching and research institutions in France or abroad, or from public or private research centers.
L'archive ouverte pluridisciplinaire HAL, est destinée au dépôt et à la diffusion de documents scientifiques de niveau recherche, publiés ou non, émanant des établissements d'enseignement et de recherche français ou étrangers, des laboratoires publics ou privés. 


\title{
Implication of epigenetics in pancreas development and disease
}

\author{
Evans Quilichini, PhD Student ${ }^{\text {a,b }}$ \\ Cécile Haumaitre, PhD, Principal Investigator ${ }^{\text {a, b, } \mathbf{c}^{*}}$
}

\author{
${ }^{a}$ Centre National de la Recherche Scientifique (CNRS), UMR7622, Institut de Biologie Paris-Seine (IBPS), \\ Paris F-75005, France. \\ ${ }^{\mathrm{b}}$ Sorbonne Universités, UPMC Université Paris 06, UMR7622-IBPS, Paris F-75005, \\ France. \\ ${ }^{\mathrm{c}}$ Institut National de la Santé et de la Recherche Médicale (INSERM), France. \\ * Corresponding author. UMR 7622, CNRS, UPMC, IBPS, Department of Developmental Biology. 9 quai Saint \\ Bernard, 75005 PARIS, FRANCE. \\ Tel.: 331442721 51; Fax: 33144273445 . \\ E-mail address: cecile.haumaitre@inserm.fr (C. Haumaitre).
}

Pancreas development is controlled by a complex interaction of signaling pathways and transcription factor networks that determine pancreatic specification and differentiation of exocrine and endocrine cells. Epigenetics adds a new layer of gene regulation. DNA methylation, histone modifications and non-coding RNAs recently appeared as important epigenetic factors regulating pancreas development. In this review, we report recent findings obtained by analyses in model organisms as well as genome-wide approaches that demonstrate the role of these epigenetic regulators in the control of exocrine and endocrine cell differentiation, identity, function, proliferation and regeneration. We also highlight how altered epigenetic processes contribute to pancreatic disorders: diabetes and pancreatic cancer. Uncovering these epigenetic events can help to better understand these diseases, provide novel therapeutical targets for their treatment, and improve cell-based therapies for diabetes.

Keywords

- $\quad$ epigenetics;

- pancreas development;

- $\beta$-cells;

- DNA methylation;

- histone modifications;

- non-coding RNAs;

- $\quad$ stem cell differentiation;

- diabetes;

- pancreatic cancer 


\section{Introduction}

The pancreas originates from the embryonic foregut endoderm. Pancreas development is based on a series of lineage decisions, giving rise to endocrine and exocrine cells. The exocrine pancreas consists of acinar cells that secrete digestive enzymes into the ductal network. Islets of Langerhans containing hormone-producing endocrine cells, including $\beta$ (insulin), $\alpha$ (glucagon) and $\delta$ (somatostatin) cells, maintain glucose homeostasis. Transcription factors (TFs) play dominant roles in instructing this differentiation program, such as Pdx 1 required for the specification of multipotent pancreatic progenitor cells (MPCs), and Neurog3 specifying endocrine precursors (reviewed in Ref. [1]). However, less is known about the epigenetic mechanisms that coordinately regulate pancreatic lineage determination and cell-fate decisions.

Epigenetics refers to heritable changes in gene expression patterns without alteration of the underlying DNA sequence. DNA methylation, histone modifications and non-coding RNAs (ncRNAs) are currently considered as epigenetic mechanisms. (i) DNA methylation occurs at cytosine-guanine (CpG) sites and is associated with gene silencing in a cell-type-dependent manner. The DNA methylation profile is established and maintained by DNA methyltransferases (DNMTs): Dnmt1, Dnmt3a and Dnmt3b, and changes dynamically during development [2]. (ii) Post-translational modifications of histones modulate chromatin structure and regulate accessibility of genes to TFs by formation of permissive or repressive states. Histone-modifying enzymes promote the addition or the loss of specific marks such as methylation, acetylation or ubiquitination. Histone methylation is catalyzed by histone methyltransferases (HMTs), whereas the reverse reaction is promoted by histone demethylases (HDMs). Polycomb-group (PcG) proteins form two families of complexes, Polycomb-repressive complex 1 (PRC1) and PRC2, and establish key repressive histone methylation marks. PRC2 comprises Ezh2, which catalyzes the trimethylation of histone $\mathrm{H} 3$ lysine 27 (H3K27me3), a repressive histone mark. PRC1 includes Bmi1 and Ring1b, catalyzing H2AK119 ubiquitination. Histone methylation can also be associated with transcriptional activation. H3K4me3, catalyzed by Trithorax-group (TrxG) proteins, is a prominent active histone mark [3]. Histone acetylation, mediated by histone acetyltransferases (HATs), prevents chromatin condensation, thus allowing transcriptional activation. Conversely, removal of acetyl groups leads to chromatin compaction resulting in transcriptional repression. Deacetylation is mediated by histone deacetylases (HDACs), which are grouped into different classes such as class I (Hdac1, -2, -3, -8) and class IIa (Hdac4, -5, -7, -9) [4]. (iii) NcRNAs refer to a large cluster of RNAs which are not translated into proteins. They include microRNAs (miRNAs, <20 nucleotides) and long ncRNAs (lncRNAs, >200 nucleotides), whose expression is tissue and stage specific. MiRNAs alter mRNA stability, and even though they are not considered a true epigenetic mechanism, they interact with epigenetic regulators such as Dnmt3, Ezh2 and Hdac1. Long ncRNAs bind to TFs and recruit chromatin-modifying complexes [5].

In this review, we first present implication of these epigenetic factors along the steps of pancreas development. Then, we depict the epigenetic mechanisms ensuring maintenance of pancreatic cell identity, function and regeneration. We discuss epigenetic regulation of stem cell differentiation and reprogramming. Finally, we highlight importance of epigenetics in disease: diabetes and pancreatic cancer. For each part, DNA methylation, histone-modifications and ncRNAs are described sequentially. Implication of epigenetics in pancreas development, function and disease is illustrated in Fig. 1. The main epigenetic regulators are summarized in Table 1. 
Fig. 1. Implication of epigenetics in pancreas development, function and disease.

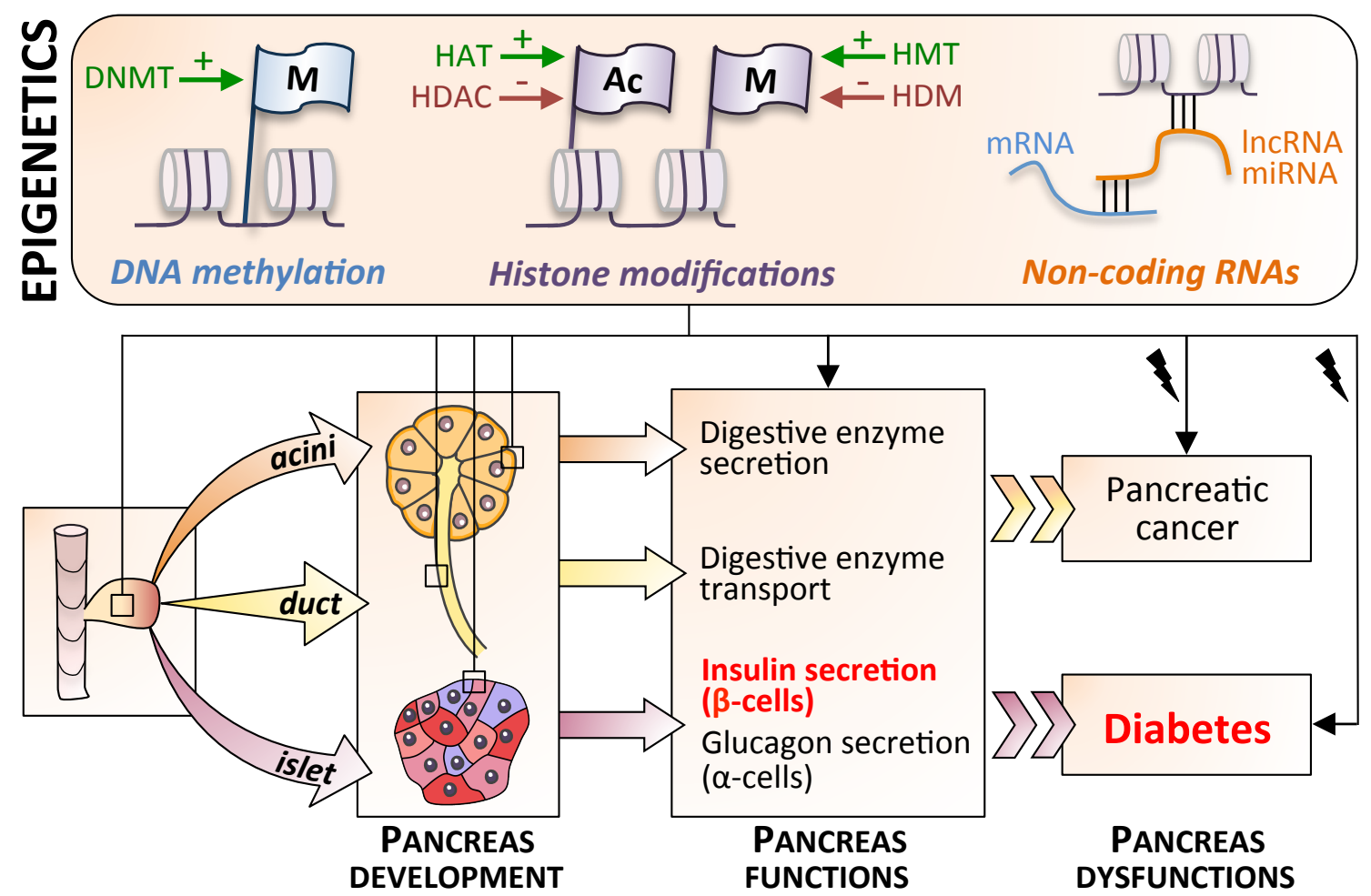

Table 1. Main epigenetic regulators cited in the text.

\begin{tabular}{|c|c|c|c|}
\hline Epigenetic mechanism & Epigenetic mark & Regulated by & Function \\
\hline \multicolumn{4}{|l|}{ DNA methylation } \\
\hline DNA methylation & / & DNA methyltransferases (DNMTs): DNMT1, DNMT3A, DNMT3B & Repression \\
\hline DNA demethylation & / & TET family proteins & Activation \\
\hline \multicolumn{4}{|l|}{ Histone modifications } \\
\hline Histone methylation & H3K27me3 & $\begin{array}{l}\text { Polycomb-Repressive Complexes (PRC1 and PRC2): } \\
\text { Histone methyltransferase (HMT) EZH2 (in PRC2) }\end{array}$ & Repression \\
\hline Histone ubiquitination & H2AK119ub1 & BMI and RING1B (in PRC1) & Repression \\
\hline \multirow{2}{*}{ Histone methylation } & H3K4me1/me2 & HMT SET7/9 & Activation \\
\hline & H3K4me3 & Trithorax-group (TrxG) proteins: HMT MLL1 & Activation \\
\hline Histone demethylation & $\mathrm{H} 3 \mathrm{~K} 27$ & Histone demethylases (HDMs): KDM6A (UTX) and KDM6B (JMJ3) & Activation \\
\hline Histone acetylation & H3K4ac/H3K27ac & Histone acetyltransferases (HATs) & Activation \\
\hline Histone deacetylation & / & Histone deacetylases (HDACs) & Repression \\
\hline \multicolumn{4}{|l|}{ Chromatin remodeling } \\
\hline Nucleosome remodeling & / & SWI/SNF Complex: BRG1 and BRM & Activ./Repress. \\
\hline \multicolumn{4}{|l|}{ Non-coding RNAs } \\
\hline miRNA & / & Endonuclease Dicer (governs miRNA processing) & Activ./Repress. \\
\hline
\end{tabular}




\title{
Epigenetics of pancreas development
}

\author{
Epigenetic mechanisms regulating differentiation of embryonic stem (ES) cells \\ to multipotent pancreatic progenitor cells (MPCs)
}

Chromatin reorganization controls accessibility for TFs and prepare ES cells to respond to signals, thus allowing differentiation along a particular lineage, such as the endoderm. DNA methylation ensures the proper spatial and temporal developmental gene regulation by TFs contributing to the restriction of the differentiation potential. As an example, loss of DNA methylation allowed the conversion of mesoderm cells to endoderm cells in response to the TF Gata4 in Dnmt1- and Dnmt3a/Dnmt3b-deficient mesoderm cells [6].

Genome-wide studies have revealed that the promoters of lineage regulators in ES cells contain bivalent chromatin domains that harbor both repressive (H3K27me3) and active (H3K4me3) marks. This bivalent chromatin profile keeps lineage regulators poised for rapid activation and plays important roles in maintaining the balance between self-renewal and differentiation. In response to differentiation cues, the bivalent domains are resolved with the removal of $\mathrm{H} 3 \mathrm{~K} 27 \mathrm{me} 3$ and activation of lineage-specific developmental genes, by the HDMs Kdm6a (Utx) and Kdm6b (Jmjd3) that play an important role in endoderm differentiation [7]. Kdm6b sequentially associates with two T-box factors, Tbx3 and Eomes, leading to a spatial reorganization of the chromatin at the Eomes locus and response to Activin signaling, thus driving ES cell differentiation towards the definitive endoderm lineage [8]. Kdm6a and Kdm6b also contribute to definitive endoderm differentiation through modulation of the WNT pathway, by sequentially activating Wnt3 and Dkk1 [9]. Liver and pancreas progenitors arise from foregut endoderm and their regulatory elements exhibit distinct functional pre-pattern of chromatin states in undifferentiated endoderm cells. The H3K27me3 repressive mark was enriched at the pancreas elements such as Pdx1, by contrast with the liver elements. The H3K27 methyltransferase Ezh2 indirectly promotes the liver program by restraining the differentiation of endoderm cells into the pancreatic lineage. In parallel, a pathway by which the BMP signal recruits the HAT P300 results in histone acetylation at liver target elements and enhances liver bud emergence [10]. Hdac1 mediates also a fate switch, being required for establishing hepatic and exocrine pancreatic fates at the expense of non-hepatic foregut endoderm in zebrafish [11].

Epigenetic regulation occurs at the stage of MPCs. Pancreas development is abrogated when Dnmt1 is deleted from MPCs. Dnmt1 represses the p53 regulatory region to maintain MPC survival [12]. Another study investigated the influence of the Swi/Snf chromatin remodeler, composed of the two core ATPase subunits, Brg1 and Brm [13]. Conditional inactivation of Brgl in MPCs leads to a normal but hypoplastic pancreas. Brg1 probably decreases $\mathrm{Pdx} 1^{+}$MPC number by affecting their proliferative capacity, through Pdx 1 recruitment of Swi/Snf to progenitor target genes [14]. The RNAi machinery has also a role in maintaining MPCs, as conditional inactivation of the miRNA processing enzyme Dicerl in MPCs leads to pancreas agenesis and neonatal death [15].

\section{Epigenetic mechanisms regulating differentiation of MPCs to exocrine cells}

The role of DNA methylation in exocrine cell differentiation was assessed by knockdown of Dnmt1 in zebrafish embryos, showing that Dnmt1 is required for acinar cell survival but not for pancreatic duct or endocrine cell formation [16] and [17].

HDACs are involved in acinar cell differentiation. Treatment of embryonic pancreas explants with HDAC inhibitors (HDACi) suppresses acinar differentiation, whereas ductal and endocrine cell fates are promoted. This demonstrates that modulation of acetylation by HDAC inhibition regulates pancreatic cell fate decision from MPCs [18]. Hdac1 is required for exocrine differentiation in zebrafish [11]. Hdac1 plays a proliferative role in acinar and ductal morphogenesis, mediating cell cycle progression through modulation of the expression of cyclin-dependant kinase (CDK) inhibitors and the SHH pathway components [19].

MiRNAs also control acinar cell differentiation. Conditional inactivation of Dicer in acinar cells has shown that miRNAs are required to establish acinar cell differentiation, and maintain acinar cell morphology and polarity. Let-7b and miR-495 repress the TF Hnf6, constituting a critical network that ensure acinar homeostasis, by driving differentiation of acinar cells while repressing hepatic gene expression [20]. MiR-18a plays also a finetuning role in acinar cell differentiation, by regulating expression of the TF Ptf1a in MPCs and acinar cells [21]. 


\section{Epigenetic mechanisms regulating differentiation of MPCs to endocrine cells}

A genome-wide analysis of the repressive mark H3K27me3 was performed during pancreatic endocrine specification, by comparing endoderm cells, pancreas progenitors, and endocrine progenitors from mouse embryos at different stages. H3K27me3 domains increase in number during endocrine progenitor development in vivo. In correlation, Ezh2 that catalyzes $\mathrm{H} 3 \mathrm{~K} 27 \mathrm{me} 3$ restrains the induction of the endocrine commitment step, as deletion of $E z h 2$ at the pancreas progenitor stage enhances the production of endocrine progenitors and $\beta$ cells. Moreover, inhibition of Ezh2 activity in embryonic pancreas explants increases endocrine progenitors [22]. Ring $1 \mathrm{~b}$ establishes repressed states that refine the neuroendocrine phenotype of islets and prevent inappropriate gene expression in differentiated cells. Ringlb bookmarks selected target genes in MPCs to establish transcriptional repression of genes that are later locked in a stably repressed state through Ring $1 \mathrm{~b}$-independent mechanisms in differentiated $\beta$-cells [23].

Endocrine differentiation is also controlled by HDACs, since inhibiting HDAC enhances endocrine differentiation. Treatment of embryonic pancreas explants with HDACi promotes the Neurog3 pro-endocrine lineage, leading to an increased pool of endocrine progenitors. Moreover, TSA and NaB treatment increases $\beta$ cells [18] and [24]. The role of class IIa HDACs was explored and their expression was found restricted to $\beta$ and $\delta$-cells. An increased $\beta$-cell and/or $\delta$-cell mass was found in $H d a c 4,-5$ and -9 mutant pancreases. Treatment of pancreatic explants with the class IIa HDACi MC1568 enhances expression of Pax4, a key TF required for proper $\beta$ - and $\delta$-cell differentiation, and amplifies the pool of $\beta$ - and $\delta$-cells. Conversely, Hdac 4 and Hdac5 overexpression shows a decreased pool of $\beta$ - and $\delta$-cells. Thus, a dynamic change in histone acetylation is important for the specification of the different endocrine cell subtypes and Hdac4, -5, -9 are key epigenetic regulators of $\beta$ - and $\delta$-cell differentiation [25]. Another epigenetic complex, composed of the TF Nkx2.2, Dnmt3a and the repressor Grg3, regulates endocrine cell subtype differentiation. At embryonic day 13.5 (E13.5), Grg3-mediated Nkx2.2 repressor activity influences the competency of the Neurog $3^{+}$endocrine progenitor to differentiate into $\varepsilon$ - versus $\beta$-cells [26].

MiRNAs are required for endocrine cell maturation. Conditional deletion of Dicer in MPCs causes a loss of endocrine cells, attributed to a reduced number of Neurog3+ cells [15]. Deletion of Dicer in endocrine progenitors leads to islet morphological defects in the neonatal period, loss of insulin expression and diabetes [27]. A role for miR-375 was determined in pancreatic islet development in zebrafish [28]. MiR-7, specifically expressed in endocrine precursors and in mature endocrine cells, acts to limit Pax6 expression, a TF pivotal in hormone-producing cell type differentiation, allowing precise endocrine cell maturation [29]. A study highlighted a link between miRNA, DNA demethylation, and endocrine cell differentiation. MiR-26a is able to directly target Ten eleven translocation (TET) enzymes, recently identified to mediate DNA demethylation, and then modulates 5-hydroxymethylcytosine levels $(5 \mathrm{hmC})$. Regulation of TETs by miR-26a promotes differentiation and proliferation of endocrine cells. TETs are downregulated in islets during postnatal differentiation, whereas miR-26a is upregulated. Overexpression of miR-26a in mice increases postnatal islet cell number [30]. LncRNAs appear also to have a role in islet maturation as more than one thousand lncNRAs were found conserved in mouse and human islets. Remarkably, 55\% of identified lnRNAs were islet specific and developmentally regulated $*[31]$ and [32].

\section{Epigenetics and pancreas plasticity}

\section{Epigenetic mechanisms regulating maintenance of exocrine cell identity and regeneration}

Epigenetic mechanisms are involved in the morphogenetic plasticity of exocrine cells, essential in differentiation and tumorigenesis processes. Acinar cells display a robust capacity to undergo regeneration and renewal in response to insults that disrupt tissue integrity. Pancreatic injury triggers a regenerative program, which is crucial for pancreas recovery. This program is critical for the maintenance of tissue homeostasis as sustained engagement of tissue repair mechanisms is linked to neoplasia. Loss of acinar cell identity during pancreatic injury leads to acinar-to-ductal metaplasia (ADM). Chronic exposure to injury and regeneration in the pancreas is associated with high neoplastic risk. The process of regeneration associated with acute or chronic pancreatic injury involves an induction of Bmil expression in acinar cells, this member of the PRC1 complex being normally not expressed in adult acinar cells. Moreover, Bmi1 plays a role in preneoplastic states in the pancreas and in tumor development. In areas of chronic pancreatic damage, Bmil expression correlates with increased cell 
proliferation and ADM [33]. Ezh2 is required for acinar cell regeneration. Ezh2 is upregulated following pancreatic injury and conditional inativation of Ezh2 in acinar cells results in a defective regenerative response to cerulein-induced injury in mice, with persistence of metaplastic lesions reflecting their compromised proliferation. Ezh2 is indeed required to maintain gene silencing of the $p 16^{I N K 4 A}$ cell cycle inhibitor in the metaplastic epithelium of the regenerating pancreas. Ezh2 restricts neoplastic progression through homeostatic mechanisms that control regeneration and prevent inflammation [34].

\section{Epigenetic mechanisms regulating endocrine cell identity and function}

Islet cells display a cell-type epigenomic plasticity. A human cell-type specific study of histone methylation signatures, by ChIP-sequencing and RNA-sequencing, determined the epigenetic and transcriptional landscape of $\alpha$ - and $\beta$-cells. In contrast to $\beta$-cells, differentiated $\alpha$-cells exhibit many genes marked bivalently (with activating H3K4me3 and repressing H3K27me3 marks), and a cell-fate conversion of $\alpha$ - to $\beta$-cells can be promoted by manipulating the histone methylation signature of human islets [35]. In differentiated $\beta$-cells, the epigenetic landscape needs to be actively maintained to stabilize cellular identity and DNA methylation is essential for repression of $\alpha$-cell lineage genes. An active repression of Arx, a major regulator of $\alpha$-cell, is required to maintain $\beta$-cell identity. Deletion of Dnmt1 or Dnmt3a leads to derepression of Arx, and $\beta$ - to $\alpha$-cell conversion. In $\beta$-cells, Nkx2.2 binds the hypermethylated promoter of Arx, in a complex with Dnmt3a and preferentially recruits Grg3 and Hdac1 to repress Arx transcription [26]. In addition, methylated region of the Arx locus in $\beta$-cells is bound by the methyl-binding protein MeCP2, which recruits the HMT PRMT6, that mediates histone arginine H3R2 methylation, resulting in repression of Arx [36]. Altogether, these studies show that, in $\beta$-cells, Nkx2.2, Dnmt3a, and MeCP2 coordinately bind the methylated DNA of the Arx promoter, recruit the histone modifiers HDAC1 and PRMT6, and repress Arx expression, illustrating the importance of epigenetic factors in maintaining adult $\beta$-cell identity.

TFs such as Pdx1 have important role in mature $\beta$-cell function and need chromatin-modifying factors to modulate gene transcription effectively. By the differential recruitment of HAT or HDAC, Pdx 1 provides an epigenetic dynamic regulation of Insulin transcription in response to glucose. In $\beta$-cell lines, the HAT P300 cooperates with $\mathrm{Pdx} 1$ to facilitate Insulin transcription under high-stimulating glucose concentrations. Conversely, under low-inhibitory glucose levels, Pdx1 associates with Hdac1 and Hdac2 to inhibit Insulin transcription [37], [38] and [39]. Blood glucose signaling also regulates dynamically the association of Pdx 1 to the Swi/Snf chromatin remodeler. The antagonistic actions of Brg1-Swi/Snf and Brm-Swi/Snf distinctly affect target gene expression in $\beta$-cells and have significant implications for glucose homeostasis. Brg1-Swi/Snf serves as a coactivator of Pdx1-mediated gene expression in $\beta$-cells, whereas Brm-Swi/Snf acts in a corepressive manner [14]. Strongly enriched in islets, the HMT Set7/9 is necessary for the transcription of essential genes controlling glucose-stimulated insulin secretion in primary islets. Set7/9 is recruited to specific $\beta$-cell genes such as Insulin and Glut2 through interaction with TFs such as Pdx1, leading to maintenance of the active mark H3K4me2 [40]. Under conditions of hyperglycemia and endoplasmic reticulum stress, Set7/9 displays nuclearto-cytoplasmic localization in $\beta$-cells. Exclusion of Set7/9 from the nucleus correlated with a loss of H3K4me2 at the Insulin promoter and a decreased $\beta$-cell secretory function [41].

MiRNAs are required in $\beta$-cells by controlling expression levels of genes essential for maintenance of $\beta$-cell identity and function (reviewed in [42]). A $\beta$-cell specific conditional inactivation of Dicer, resulting in a global loss of miRNAs, leads to progressive impairment of insulin secretion, glucose homeostasis and diabetes [43]. The profile of miRNAs differentially expressed in $\alpha$ - and $\beta$-cells was determined, as well as their potential TF targets [44]. Among them, miR-375 is a critical regulator of $\beta$-cell function [45], miR-26, miR-148 and miR-182 regulate Insulin promoter activity [46]. LncRNAs seem also to contribute to the unique regulatory environment found in islet cell-subtypes. The study that evaluated the cell-specific epigenetic and transcriptional landscape of human $\alpha$ - and $\beta$-cells identified $12 \beta$-cell-specific and $5 \alpha$-cell-specific lncRNAs [35]. LncRNAs appear to control $\beta$-cell function through the regulation of imprinted loci [47]. A $\beta$-cell-specific lncRNA, the lncRNA $H I$ $L N C 25$, influences gene expression in mature $\beta$-cells. Depletion of HI-LNC25 indeed decreases expression of the TF and monogenic diabetes gene GLIS3 [31].

\section{Epigenetic mechanisms regulating $\beta$-cell proliferation and regeneration}

Regulation of $\beta$-cell proliferation is critical to adapt to metabolic demand. For example, Menin-dependent histone methylation provides an epigenetic mechanism for controlling $\beta$-cell expansion during pregnancy. In pregnant mice, the hormone Prolactin reduces levels of Menin, an endocrine tumor suppressor and 
transcriptional regulator. Menin associates with a HMT complex, promoting H3K4me3. This maintains expression of $p 27^{K i p l}$ and $p 18^{I N K 4 C}$, which encode CDK inhibitors and prevent islet proliferation [48].

$\beta$-cell proliferation and regeneration decrease with age and are regulated by epigenetic modifications at the $C d k n 2 a$ locus through PRC1, PRC2 and TrxG complexes. Expression of the cell cycle inhibitor p16 ${ }^{\mathrm{INK} 4 \mathrm{a}}$, encoded by $C d k n 2 a$, increases with age, and reduces the proliferative and regenerative capacity of aged $\beta$-cells. Ezh2 promotes $\beta$-cell replication, by repressing $C d k n 2 a$, through $\mathrm{H} 3 \mathrm{~K} 27 \mathrm{me} 3$. With age, there is a decreased expression of the two PcG proteins Ezh2 and Bmi1. This is associated with a loss of repressive marks, essentially $\mathrm{H} 3 \mathrm{~K} 27 \mathrm{me} 3$, decreased Bmi1 binding, loss of H2A ubiquitination, increased recruitment of the TrxG protein Mll1, and a concomitant increase in $\mathrm{H} 3 \mathrm{~K} 4 \mathrm{me} 3$, leading to derepression of the Cdkn2a locus [49] and [50]. During regeneration, after $\beta$-cell destruction with streptozotocin, increased Bmil binding to the $C d k n 2 a$ locus coincides with decreased $\mathrm{H} 3 \mathrm{~K} 4 \mathrm{me} 3$ and leads to a decrease in p16 ${ }^{\text {Ink4a }}$ levels, resulting in increased $\beta$-cell proliferation [50]. In old mice, combined knockdown of Mll1-containing TrxG complex with Ezh2 overexpression increases $\beta$-cell replication [51]. Thus, epigenetic manipulation of these complexes or their respective histone modification marks in $\beta$-cells might be a useful tool for promoting $\beta$-cell proliferation and regeneration.

\section{Epigenetics of embryonic stem cell differentiation and reprogramming}

In clinical practice, type 1 diabetic patient can receive islet transplantation of two or three donors, but the scarcity of islet donors has restricted this therapy. A major goal is to generate unlimited number of $\beta$-cells from ES cells (ESC) or human induced pluripotent stem cell (hPSC), as a renewable cell-based therapy for the treatment of diabetic patients. Several protocols based on the fundamental knowledge acquired by studying pancreatic development attempted to recapitulate these different steps from ESC to definitive endoderm (DE), primitive gut tube (GT), pancreatic endoderm (PE), endocrine precursors and $\beta$-cells. Epigenetic compounds and compounds that regulate signaling pathways help to ameliorate these protocols to produce functional $\beta$-cells at a sufficiently high efficiency [52], [53] and [54]. The majority of protocols have failed to produce fully functional $\beta$-cells without an in vivo maturation stage in mice. Thus, understanding regulators such as epigenetic actors involved in this critical maturation stage is crucial for the therapeutic generation of $\beta$-cells. For example, the six lncRNAs whose expression was found only activated at this last stage could be useful *[31] and [32]. One identified dysfunction of in vitro-produced $\beta$-cells is an inappropriate remodeled chromatin compared to primary human islets, linked to a failure to eliminate PcG-mediated repression on endocrine-specific genes, thus identifying a possible strategy for improving cell differentiation [55]. Finally, precise modifications of epigenetic profiles may allow conversion of pancreatic cells into bona fide $\beta$-cells.

These protocols can also help to understand the epigenetic mechanisms that orchestrate remodeling of the chromatin landscape and drive the different steps of differentiation. Epigenetic regulation of gene enhancer elements is important for establishing cell identity. Histone H3K27ac distinguishes active enhancers from unmarked (i.e. inactive, lacking all of the features associated with the active enhancer state), or poised enhancer, carrying $\mathrm{H} 3 \mathrm{~K} 4 \mathrm{me} 1$ in the absence of $\mathrm{H} 3 \mathrm{~K} 27 \mathrm{ac}$ [56]. Poised enhancers are important for the plasticity of developmental decisions. They can acquire the signature of active enhancers in response to environmental or developmental stimuli. These regulatory events at enhancers contribute to the specific gene expression programs that determine cell state and the potential for differentiation into new cell types. They might facilitate cell reprogramming strategies for use in regenerative medicine [57] and [58]. A study mapped enhancer-related histone modifications during human ESCs differentiation toward the pancreatic fate at defined stages (from ESCs to PE) [59]. Classes of active enhancers (H3K4me1, H3K27ac) and poised enhancers (only H3K4me1) were defined. Interestingly, a poised enhancer landscape linked to genes of multiple GT-derived lineages is gained during the transition of DE to GT. Analysis of active enhancers in human islets determined that enhancers of endocrine-cell genes are pre-marked in PE, prior to terminal differentiation. This study allows the identification of a poised chromatin state at enhancers as a mechanism by which progenitor cells acquire developmental competence. These findings have also implications for differentiation protocols, as cells have to acquire the transcriptional competence to appropriately respond to instructive signals given by compounds [59].

Cellular reprogramming of somatic cells is an alternative strategy to generate $\beta$-cells. Altering chromatin modification state of a human cell may enhance conversion to insulin-secreting cells. For example, a study achieved the direct conversion of adult human skin fibroblasts into insulin-secreting cells, avoiding a stable 
pluripotent stage and transgenic modification. Adult human skin fibroblasts were exposed to a DNMTi followed by a three-step protocol for the induction of pancreatic endocrine differentiation [60]. In another protocol, epigenetic modulating factors (a HDACi and a DNMTi) with overexpression and silencing of genes important for pancreatic endocrine development were used on primary human dermal fibroblasts to transdifferentiate into islet-like cells that decrease glycemic levels in diabetic mice [61].

A major aspect of cellular reprogramming concerns the stability of epigenetic information, which is relevant to the functionality and safety of reprogrammed hPSC. hPSC showed a potentially high degree of imprint instability during reprogramming due to epigenetic alterations related to DNA methylation and regulation of imprinted genes [62]. Further studies will be required to resolve this question.

\section{Epigenetic mechanisms involved in diabetes}

Type 2 diabetes (T2D) is associated with a reduction in $\beta$-cell mass and impaired $\beta$-cell function. Genome-wide association studies (GWAS) have identified more than 70 loci robustly associated with T2D risk, but that explain only $\sim 11 \%$ of T2D cases. Most of the causal single nucleotide polymorphisms (SNPs) fall outside coding genes, suggesting that epigenetics is important for the pathophysiology of T2D [63].

Environment can affect gene expression through epigenetics. Animal models of prenatal undernutrition showed that rats exposed to intrauterine growth retardation (IUGR) develop T2D in the adulthood due to a selfpropagating epigenetic silencing cycle of the $P d x 1$ locus. In this model, $P d x 1$ expression is decreased due to histone deacetylation and chromatin remodeling. Hdac1 is recruited and decreases histone acetylation. The recruitment of a HMT increases $\mathrm{H} 3 \mathrm{~K} 9 \mathrm{me} 2$, repressing $P d x 1$. The phenomenon is reversible in neonatal islets with HDACi treatment but not in adult, where DNA methylation locks the promoter into a silenced state, resulting in diabetes [64]. The TF and monogenic diabetes gene $H n f 4 a$ is epigenetically regulated by maternal diet and aging in rat islets. A progressive epigenetic silencing of the entire Hnf4a locus in islets prevents enhancer-promoter interaction and increases the risk of T2D [65]. Tissue-specific methylation and parental imprinting of the growth factor receptor-bound protein 10 (GRB10) gene, a negative regulator of insulin signaling, influence glucose metabolism and contribute to T2D. Interestingly, allelic imbalance was observed with different effects on glucose metabolism, if the allele of the GRB10 variant was transmitted from fathers or mothers [66].

Altered DNA methylation at promoters of genes critical for $\beta$-cell function contributes to T2D. Increased DNA methylation at promoters of PDX1 and PPARGC1A associates with decreased gene expression and reduced insulin secretion in islets from T2D patients [67] and [68]. Global DNA methylation and gene expression analyses from islets from T2D and non-diabetic donors reveal that $96 \%$ of the sites showing differential methylation in T2D islets display decreased methylation, including genes related to signaling pathways essential for $\beta$-cell adaptation and apoptosis, such as CDK5R1 (Cyclin-dependent kinase 5 regulatory subunit 1). It encodes a direct activator of CDK5, leading to decreased transcription of PdxI and Insulin. By contrast, a minority of genes are hypermethylated and silenced in islets from T2D donors, such as PAX4, essential for $\beta$ cells. Aberrant methylation contributes to a loss of $\beta$-cell identity under pathophysiological conditions [69]. Another recent study described the human methylome and transcriptome in islets from T2D donors, providing a detailed map of the global DNA methylation pattern in islets, $\beta$ - and $\alpha$-cells. $75 \%$ of the differentially expressed genes show a decreased methylation, associated with increased gene expression in T2D islets. Candidate genes affecting $\beta$ - and $\alpha$-cells were identified, such as $C D K N 1 A$ and $E X O C 3 L 2$, which regulate $\beta$-cell proliferation and exocytosis, respectively [70]. SNPs identified as T2D risk variants might introduce or remove a CpG dinucleotide, a mechanism through which they can influence DNA methylation patterns, thus affecting gene function and contributing to the disease [71]. TFs interact functionally with the epigenome and dysregulation of islet enhancers is relevant to the mechanisms underlying T2D. Many variants associated with T2D reside in clusters of enhancers that form physical three-dimensional chromatin domains and abolish enhancer activity [72].

The epigenetic architecture of human islets was analyzed by ChIP-seq to create genome-wide maps of histone modifications associated with gene activation (H3K4me1, H3K4me2, H3K4me3, H3K79me2) and repression (H3K27me3). These epigenomic maps can serve to understand the epigenetic basis of T2D [73] and [74]. A study compared the histone methylation profile of purified $\beta$-cells to the one of MPCs, and differentiated cells including acinar, liver and neural tissue. An important epigenetic event consists in a selective removal of the repressive mark $\mathrm{H} 3 \mathrm{~K} 27 \mathrm{me} 3$ in a core developmental program that enables $\beta$-cells to acquire a neural gene 
activity state. The finding that a dynamic regulation of PcG repression program shapes the identity of $\beta$-cells provides a baseline to investigate how perturbations of the $\beta$-cell epigenome can contribute to the development of T2D [75].

MiRNAs and their epigenetic control by DNA methylation contribute to the pathogenesis of T2D. A cluster of miRNAs in the imprinted locus DLK1-MEG3 is specifically expressed in human $\beta$-cells. Downregulation of this miRNA cluster observed in T2D islets from donors correlates with hypermethylation of its promoter. The ncRNA gene MEG3 is also downregulated in T2D islets [76]. Furthermore, a miR-7 genomic circuit was shown to regulate insulin granule exocytosis in $\beta$-cells. MiR-7a levels were found decreased in diabetic mouse models and in T2D islets [77]. A decrease in miR-30d expression is also observed in the islets of diabetic mice. MiR$30 \mathrm{~d}$ protects cells from TNF- $\alpha$ suppression of Insulin transcription [78]. MiR-185 is downregulated in the islets of diabetic patients and mice. MiR-185 promotes insulin secretion and $\beta$-cell growth by targeting SOCS3 and regulating Stat3 pathway [79]. Islet enriched miRNAs could serve as blood marker to detect $\beta$-cell death and predict diabetes. The levels of circulating miR-101, miR-375 and miR-802 are increased in T2D patients and may become new biomarkers for T2D [80] and [81]. Several lncRNAs are found dysregulated in islets from T2D subjects, and one lncRNA regulates GLIS3, showing that lncRNAs have the ability to control important diabetes genes [31]. The fact that lncRNAs have the potential to regulate gene expression and cellular identity in $\beta$-cells offers the opportunity to better understand T2D etiology and novel targets for treatment [47] and [82].

\section{Epigenetic mechanisms involved in pancreatic cancer}

In up to $95 \%$ cases, pancreatic cancer arises in exocrine cells, the most common being pancreatic ductal adenocarcinoma (PDAC). It arises from precursor lesions, including pancreatic intraepithelial neoplasia (PanIN) and intraductal papillary mucinous neoplasia (IPMN) [83]. The role of Kras as an initiating cancer mutation is one of the best established mechanism required for the development of pancreatic cancer. In addition to an accumulation of somatic mutations that affect the DNA sequence directly, epigenetic reprogramming can lead to an increased cell growth and survival, and contribute to tumorigenesis.

DNA methylation of tumor suppressor gene promoter sites is a major epigenetic mechanism, which plays a role in tumorigenesis. APC, BRCA1, p16 ${ }^{I N K 4 a}$ are the most frequently methylated gene promoter regions in human pancreatic neoplasms [84]. Genome-wide patterns of DNA methylation in PDAC reveal an enrichment of aberrantly methylated genes involved in key mechanisms: TGF- $\beta$, WNT, integrin signaling, cell adhesion, stellate cell activation. Another analysis suggests an epigenetic suppression of SLIT-ROBO signaling and upregulation of MET and ITGA2 [85]. Epigenetic silencing of EYA2, associated with promoter methylation and histone deacetylation, promotes tumor growth, whereas overexpression in mice limits the growth and metastases of PDAC [86]. Downregulation of WNK2 by promoter hypermethylation is observed in PanIN and in a PDAC mouse model, enhancing tumor cell growth via the ERK-MAPK pathway [87]. Aberrant DNA methylation of miRNAs occurs in PDAC. MiR-615-5p is silenced in PDAC cell lines by promoter hypermethylation, which limited its inhibition on $I G F 2$, thereby contributing to tumor growth and invasion [88]. PDAC have patterns of gene methylation that differ from pancreatic endocrine tumors (pNET). Analysis of the epigenome of pNET tissue also identified mutations of the chromatin-remodeling genes $A T R X / D A X X$ and inactivating mutations of MEN1, encoding menin [89].

PDAC involves an increased expression of DNMT1, DNMT3A and DNMT3B. A cooperation between DNMT1 and HDAC1 was suggested, DNA methylation initiating the repression, which is then executed by histone deacetylation. Increased expression levels of DNMT1 and HDACl are found from precancerous lesions to pancreatic cancer, and reflect the malignancy of PDAC [90]. HDAC1 contributes to the uncontrolled proliferation during pancreatic tumorigenesis. The developmental events described above, in which Hdac1 mediates cell cycle progression through modulation of the expression of CDK inhibitors and the SHH pathway components, are recapitulated in human PDAC cells [19]. Expression of Sin3b, encoding proteins of the Hdac1/2 repression complex, was also found upregulated in a mouse model of PDAC [91]. Sin3b inactivation in mice leads to delayed PDAC progression, associated with impaired inflammatory response [92]. Moreover, $\operatorname{Sin} 3 b$ was found as a novel direct target of Bmil forming an essential epigenetic complex of cellular senescence. Upon oncogenic stress, Bmil dissociates from the $\operatorname{Sin} 3 b$ locus, resulting in increased $\operatorname{Sin} 3 b$ expression and entry into cellular senescence through derepression of the $C d k n 2$ locus [93]. Brg1 is also a critical epigenetic regulator of genes important for IPMN and PDAC progression. Acinar deletion of Brgl in mice cooperates with oncogenic Kras to form cystic neoplastic lesions that resemble human IPMN and progress to PDAC. Enrichment for $\mathrm{H} 3 \mathrm{~K} 27 \mathrm{Me} 3$ mark on the promoters of several genes is associated with reduced expression of these genes in 
Brg1-depleted IPMN-PDAC cells [13]. Methyl-CpG binding domain protein 1 (MBD1) promotes pancreatic cell invasion. MBD1 forms a complex with Twist and NAD-dependent deacetylase SIRT-1 on the CDH1 promoter, which results in epigenetic downregulation of E-cadherin and increased epithelial-to-mesenchymal transition [94]. Bmil, Ringlb and Ezh2 are upregulated in PDAC, and H2AK119Ub1 and H3K27Me3 marks may serve as discriminatory biomarkers for PDAC staging [33] and [95]. The genomic landscape of PDAC human cell lines, explored by ChIP-seq and RNA-seq, reveals regions with an enrichment of positive marks (H3K4me1, H3K4me3, RNA Pol II) and a depletion of negative marks (H3K27me3), in correlation with gene expression. Global analyses of epigenetic marks and transcribed sequences can contribute to understand the mechanisms underlying PDAC. In this study, $A L D H 1 A 3$ was upregulated and identified as a novel candidate gene for PDAC [96].

Misexpression of miRNAs is commonly observed in PDAC and its precursor lesions (reviewed in [97]). For example, miR-21 and miR-155 are upregulated in IPMNs and their expression correlates with histological features of progression [98]. By contrast, lower levels of miR-101 can be a trigger for the adenocarcinoma sequence of IPMN, by upregulation of EZH2 [99].

Thus, epigenetic modifications contribute to the pathogenesis of T2D and to malignant cellular transformation. Therefore, the use of novel targeted epigenetic therapies is very attractive, and may be promising due to the relative reversibility of DNA methylation and chromatin modifications.

\section{Practice points}

- Specific epigenetic marks can predict cell fate during development.

- Epigenetics has essential roles in pancreatic cell differentiation, maintenance of identity and function.

- Endocrine pancreas displays an inter-cellular plasticity highlighted by bivalent epigenetic marks.

- Epigenetic modifications enhance stem cell differentiation and reprogramming efficiency to pancreatic cells.

- Alteration of pancreatic cell epigenetic landscape can induce diabetes or pancreatic cancer.

- Epigenetic regulation confers cellular plasticity to the pancreas, linked to pancreas regeneration and pancreatic diseases. Knowledge and manipulation of epigenetics can drive reprogramming of pancreatic cells, while controling the risks of cancer.

\section{Research agenda}

- Further analyses of epigenetic regulation of acinar and ductal cells

- Epigenomic maps of isolated pancreatic cell populations

- Identify epigenetic marks and epigenetic regulators that facilitate pancreatic differentiation. Target them by epigenetic editing to improve in vitro $\beta$-cell differentiation protocols from hPSC and direct reprogramming to $\beta$-cells

- Genome-wide analyses to identify non-coding variants that contribute to T2D and develop novel therapies for T2D 


\section{Summary}

The recent findings presented in this review demonstrate that epigenetic mechanisms are important regulators of pancreas development and function (summarized in Table 2). DNA methyltransferases, histone modifiers and ncRNAs regulate gene expression and, with TFs, coordinately control cell-fate decisions and the pancreatic differentiation program. These epigenetic actors drive differentiation of endoderm cells into the pancreatic lineage. Then, they are required for initiating acinar cell differentiation and maintaining acinar cell identity and homeostasis. As some embryonic mechanisms are recapitulated in the process of recovery or tumorigenesis, these epigenetic mechanisms are also involved during tissue regeneration, and can contribute to neoplasia. A repressive epigenetic state seems to be required in acinar cells, and an alteration of these epigenetic mechanisms can initiate and promote pancreatic cancer. These epigenetic actors are required for endocrine cell differentiation. Inhibition of different histone-modifying enzyme activity enhances the pro-endocrine lineage. Histone-modifying enzymes and miRNAs are required to repress a neuronal fate in endocrine cells. Finally, these epigenetic actors are required for $\beta$-cell differentiation, function, proliferation and maintenance of identity. High epigenomic plasticity allows endocrine cell-subtype reprogramming. Genome-wide analyses provide epigenomic maps that can serve as a resource for understanding the pancreatic differentiation steps and the epigenetic basis of pancreatic cancer and diabetes. These fundamental and clinical observations of epigenetic mechanisms provide new insights in the understanding of pancreas development and diseases. These epigenetic marks or actors can serve as disease biomarkers and potentially as therapeutical targets and could promote the design of new therapeutic strategies.

Table 2. Studies of the roles of epigenetic actors in pancreas development and $\beta$-cell function.

\begin{tabular}{|c|c|c|c|c|}
\hline Dev. Stage & Experiment & Cell/Organism & Phenotype & Ref \\
\hline \multirow{8}{*}{$\begin{array}{l}\text { From } \\
\text { ES cells to } \\
\text { pancreatic } \\
\text { progenitors }\end{array}$} & Dnmt3a $a^{-1}$; nnmt3 $b^{-1}$ double-knock-out & Mouse ES cells & Lineage conversion from mesoderm to endoderm & [6] \\
\hline & KDM6A or KDM6B knock-down & Human ES cells & $\begin{array}{l}\text { Impaired endoderm differentiation, rescued by sequential } \\
\text { treatment with WNT agonist and antagonist }\end{array}$ & [9] \\
\hline & FoxA3-Cre;Ezh2 $2^{f \mid f l}$ & Mouse & $\begin{array}{l}\text { Expanded pancreatic progenitor domain at the expense of liver } \\
\text { development }\end{array}$ & [10] \\
\hline & $\mathrm{P}_{300^{+/-}}$ & Mouse & Decreased specification of liver over pancreas progenitors & [10] \\
\hline & $\operatorname{Hdac1}^{5436}$ & Zebrafish & $\begin{array}{l}\text { Hepatic and exocrine pancreatic specification and differentiation } \\
\text { severely affected, expanded foregut tissue }\end{array}$ & [11] \\
\hline & Pdx1-Cre;Dnmt1 $1^{f / f l}$ & Mouse & $\begin{array}{l}\text { Pancreas agenesis due to apoptosis of pancreatic progenitor cells, } \\
\text { rescued by haploinsufficiency of } p 53\end{array}$ & [12] \\
\hline & Ptf1a-Cre;Brg1 $1^{f \mid f l}$ & Mouse & Hypoplastic pancreas & [13] \\
\hline & Pdx1-Cre;Dicer $r^{f / f l}$ & Mouse & Pancreas agenesis & [15] \\
\hline \multirow{6}{*}{$\begin{array}{l}\text { From } \\
\text { pancreatic } \\
\text { progenitors } \\
\text { to exocrine } \\
\text { cells }\end{array}$} & Dnmt1 knock-down & Zebrafish & Defects in exocrine pancreas differentiation & [16] \\
\hline & $D n m t 1^{5872}$ or Dnmt $1^{5904}$ & Zebrafish & Acinar cells apoptosis, no duct or endocrine defects & [17] \\
\hline & HDAC inhibitors & Mouse & Decreased acinar differentiation in pancreatic explants & [18] \\
\hline & $H d a c 1^{5436}$ & Zebrafish & Defects in exocrine pancreas specification and differentiation & [11] \\
\hline & Hdac1 $^{\text {hi1618 }}$ & Zebrafish & Growth arrest and dysmorphogenesis of exocrine pancreas & [19] \\
\hline & Foxa3-Cre;Dicer ${ }^{f l /-}$ or Ela-Cre;Dicer ${ }^{f l /-}$ & Mouse & Impaired acinar cell differentiation & [20] \\
\hline \multirow{13}{*}{$\begin{array}{l}\text { From } \\
\text { pancreatic } \\
\text { progenitors } \\
\text { to } \\
\text { endocrine } \\
\text { cells }\end{array}$} & Pdx1-Cre;Ezh2 $2^{f \mid f l}$ & Mouse & Increased endocrine progenitors & [22] \\
\hline & EZH2 inhibitors & $\begin{array}{l}\text { Human ES cells } \\
\text { Mouse }\end{array}$ & Increased endocrine progenitors in hESCs and pancreatic explants & [22] \\
\hline & Pdx1-Cre;Ring $1 b^{f \mid f f} /$ Ins-Cre;Ring1 $1 b^{f \mid f l}$ & Mouse & $\begin{array}{l}\text { Transcriptional derepression of a subset of direct Ring } 1 \mathrm{~b} \text { target } \\
\text { genes in differentiated islet cells / no derepression }\end{array}$ & [23] \\
\hline & HDAC inhibitors & Rat & Increased endocrine progenitors & [18] \\
\hline & $\mathrm{Hdac}^{-1-}$ & Mouse & Increased $\delta$-cells & [25] \\
\hline & $\operatorname{Hdac5}^{\%}$ & Mouse & Increased $\beta$ - and $\delta$-cells & [25] \\
\hline & $\mathrm{Hdac}^{-1-}$ & Mouse & Increased $\beta$-cells & [25] \\
\hline & Hdac4 and Hdac5 overexpression & Rat & Decreased pool of $\beta$ - and $\delta$-cells in pancreatic explants & [25] \\
\hline & $N k \times 2.2^{\text {TNmut/TNmut }}$ & Mouse & Decreased $\beta$-cells and increased $\varepsilon$-cells & [26] \\
\hline & Pdx1-Cre;Dicer ${ }^{f / f l}$ & Mouse & Impaired development of endocrine cells, especially $\beta$-cells & [15] \\
\hline & Ngn3-Cre;Dicer ${ }^{f / f l}$ & Mouse & Decreased endocrine cell mass in 2-week-old mice & [27] \\
\hline & MiR-375 knock-down & Zebrafish & Aberrant migration of pancreatic islets & [28] \\
\hline & MiR-7 overexpression & Mouse & Pax6 downregulation and inhibition of $\alpha$ - and $\beta$-cell differentiation & [29] \\
\hline
\end{tabular}




\begin{tabular}{|c|c|c|c|c|}
\hline & MiR-7 knockdown & Mouse & Pax6 upregulation and enhancement of $\alpha$ - and $\beta$-cell differentiation & [29] \\
\hline & MiR-26a overexpression & Mouse & Increased islet cell number & [30] \\
\hline \multirow{12}{*}{$\begin{array}{l}\text { Mature } \\
\beta \text {-cells }\end{array}$} & HMT inhibitor & Human islets & Reprogramming of $\alpha$ - to $\beta$-cells & [35] \\
\hline & $N k \times 2.2^{\text {TNmut/TNmut }}$ & Mouse & Ectopic expression of $A r x$ in $\beta$-cells, reprogramming of $\beta$ - to $\alpha$-cells & [26] \\
\hline & Rip-Cre;Dnmt1 ${ }^{f l / f l}$ & Mouse & Reprogramming of $\beta$-cells to $\alpha$-cells & [36] \\
\hline & $\begin{array}{l}\text { Brg1 knock-down } \\
\text { Brm knock-down }\end{array}$ & INS-1 $\beta$-cells & $\begin{array}{l}\text { Decreased Insulin, MafA and Glut2 expression } \\
\text { Increased Insulin, MafB, Glut2 and Ucn3 expression }\end{array}$ & [14] \\
\hline & Set7/9 knock-down & Mouse islets & Defects in insulin secretion & [40] \\
\hline & RIP-Cre;Dicer ${ }^{f|f|}$ & Mouse & Reduced $\beta$-cell mass and impaired glucose secretion & [43] \\
\hline & MiR-375 KO & Mouse & $\begin{array}{l}\text { Increased } \alpha \text {-cell number and decreased } \beta \text {-cell mass due to impaired } \\
\beta \text {-cell proliferation, hyperglycemia }\end{array}$ & [45] \\
\hline & MiR-26, miR-182, miR-148 knock-down & $\begin{array}{l}\text { MIN6 } \beta \text {-cells } \\
\text { Mouse islets }\end{array}$ & Decreased Insulin expression & [46] \\
\hline & RIP-rtTA;Men1 (Men1 overexpression) & Mouse & Impaired maternal $\beta$-cell expansion during pregnancy & [48] \\
\hline & RIP-Cre;Ezh2 $2^{f \mid f l}$ & Mouse & $\begin{array}{l}\text { Accelerated loss of } \mathrm{H} 3 \mathrm{~K} 27 \mathrm{me} 3 \text { repression at } C d k n 2 a \text {, premature } \\
p 16^{I N K 4 a} \text { expression, reduced } \beta \text {-cell proliferation and mass, mild } \\
\text { diabetes and impaired } \beta \text {-cell regeneration }\end{array}$ & [49] \\
\hline & $\mathrm{Bmi}^{-/}$ & Mouse & $\begin{array}{l}\text { Reduced } \beta \text {-cell proliferation in young mice and impaired } \beta \text {-cell } \\
\text { regeneration }\end{array}$ & {$[50]$} \\
\hline & RIP-rtTA;Ezh2TG (Ezh2 overexpression) & Mouse & Increased $\beta$-cell replication in young mice & [51] \\
\hline
\end{tabular}

\section{Conflict of interest}

None.

\section{Acknowledgments}

We apologize to our colleagues whose work could not be cited due to space limitations. E.Q. is recipient of a $\mathrm{PhD}$ student fellowship from the Ministère de la Recherche. C.H. is an investigator of the Institut National de la Santé et de la Recherche Médicale (INSERM). Support was received from the Centre National de la Recherche Scientifique (CNRS), the Université Pierre et Marie Curie (UPMC), the programme Emergence UPMC, the Société Francophone du Diabète (SFD)-Ypsomed, the GEFLUC (Les entreprises contre le Cancer). 


\section{References}

[1] Pan FC, Wright C. Pancreas organogenesis: from bud to plexus to gland. Dev Dyn 2011;240:530-65.

[2] Smith ZD, Meissner A. DNA methylation: roles in mammalian development. Nat Rev Genet 2013;14:20420.

[3] Schuettengruber B, Chourrout D, Vervoort M, et al. Genome regulation by polycomb and trithorax proteins. Cell 2007;128:735-45.

[4] Delcuve GP, Khan DH, Davie JR. Roles of histone deacetylases in epigenetic regulation: emerging paradigms from studies with inhibitors. Clin Epigenetics 2012;4:5.

[5] Holoch D, Moazed D. RNA-mediated epigenetic regulation of gene expression. Nat Rev Genet 2015;16:7184.

[6] Oda M, Kumaki Y, Shigeta M, et al. DNA Methylation Restricts Lineage-specific Functions of Transcription Factor Gata4 during Embryonic Stem Cell Differentiation. PLoS Genet 2013;9:e1003574.

[7] Mikkelsen TS, Ku M, Jaffe DB, et al. Genome-wide maps of chromatin state in pluripotent and lineagecommitted cells. Nature 2007;448:553-60.

[8] Kartikasari AER, Zhou JX, Kanji MS, et al. The histone demethylase Jmjd3 sequentially associates with the transcription factors Tbx3 and Eomes to drive endoderm differentiation. EMBO J 2013;32:1393-408.

[9] Jiang W, Wang J, Zhang Y. Histone H3K27me3 demethylases KDM6A and KDM6B modulate definitive endoderm differentiation from human ESCs by regulating WNT signaling pathway. Cell Res 2013;23:12230 .

* [10] Xu C-R, Cole PA, Meyers DJ, et al. Chromatin "prepattern" and histone modifiers in a fate choice for liver and pancreas. Science 2011;332:963-6.

[11] Noël ES, Casal-Sueiro A, Busch-Nentwich E, et al. Organ-specific requirements for Hdac1 in liver and pancreas formation. Dev Biol 2008;322:237-50.

* [12] Georgia S, Kanji M, Bhushan A. DNMT1 represses p53 to maintain progenitor cell survival during pancreatic organogenesis. Genes Dev 2013;27:372-7.

[13] Von Figura G, Fukuda A, Roy N, et al. The chromatin regulator Brg1 suppresses formation of intraductal papillary mucinous neoplasm and pancreatic ductal adenocarcinoma. Nat Cell Biol 2014;16:25567.

[14] McKenna B, Guo M, Reynolds A, et al. Dynamic Recruitment of Functionally Distinct Swi/Snf Chromatin Remodeling Complexes Modulates Pdx1 Activity in Islet $\beta$ Cells. Cell Rep 2015;10:2032-42.

[15] Lynn FC, Skewes-Cox P, Kosaka Y, et al. MicroRNA Expression Is Required for Pancreatic Islet Cell Genesis in the Mouse. Diabetes 2007;56:2938-45.

[16] Rai K, Nadauld LD, Chidester S, et al. Zebra Fish Dnmt1 and Suv39h1 Regulate Organ-Specific Terminal Differentiation during Development. Mol Cell Biol 2006;26:7077-85.

[17] Anderson RM, Bosch JA, Goll MG, et al. Loss of Dnmt1 catalytic activity reveals multiple roles for DNA methylation during pancreas development and regeneration. Dev Biol 2009;334:213-23.

[18] Haumaitre C, Lenoir O, Scharfmann R. Histone Deacetylase Inhibitors Modify Pancreatic Cell Fate Determination and Amplify Endocrine Progenitors. Mol Cell Biol 2008;28:6373-83.

[19] Zhou W, Liang I-C, Yee NS. Histone deacetylase 1 is required for exocrine pancreatic epithelial proliferation in development and cancer. Cancer Biol Ther 2011;11:659-70.

[20] Prévot P-P, Augereau C, Simion A, et al. Let-7b and miR-495 stimulate differentiation and prevent metaplasia of pancreatic acinar cells by repressing HNF6. Gastroenterology 2013;145:668-78.e3.

[21] Yang Y, Ding L, An Y, et al. MiR-18a regulates expression of the pancreatic transcription factor Ptf1a in pancreatic progenitor and acinar cells. FEBS Lett 2012;586:422-7.

* [22] Xu C-R, Li L-C, Donahue G, et al. Dynamics of genomic H3K27me3 domains and role of EZH2 during pancreatic endocrine specification. EMBO J 2014;33:2157-70.

[23] Van Arensbergen J, García-Hurtado J, Maestro MA, et al. Ring1b bookmarks genes in pancreatic embryonic progenitors for repression in adult $\beta$ cells. Genes Dev 2013;27:52-63.

[24] Haumaitre C, Lenoir O, Scharfmann R. Directing cell differentiation with small-molecule histone deacetylase inhibitors: the example of promoting pancreatic endocrine cells. Cell Cycle 2009;8:536-44.

[25] Lenoir O, Flosseau K, Ma FX, et al. Specific control of pancreatic endocrine $\beta$ - and $\delta$-cell mass by class IIa histone deacetylases HDAC4, HDAC5, and HDAC9. Diabetes 2011;60:2861-71.

[26] Papizan JB, Singer RA, Tschen S-I, et al. Nkx2.2 repressor complex regulates islet $\beta$-cell specification and prevents $\beta$-to- $\alpha$-cell reprogramming. Genes Dev 2011;25:2291-305.

[27] Kanji MS, Martin MG, Bhushan A. Dicer1 is required to repress neuronal fate during endocrine cell maturation. Diabetes 2013;62:1602-11.

[28] Kloosterman WP, Lagendijk AK, Ketting RF, et al. Targeted Inhibition of miRNA Maturation with Morpholinos Reveals a Role for miR-375 in Pancreatic Islet Development. PLoS Biol 2007;5. 
[29] Kredo-Russo S, Mandelbaum AD, Ness A, et al. Pancreas-enriched miRNA refines endocrine cell differentiation. Development 2012;139:3021-31.

[30] Fu X, Jin L, Wang X, et al. MicroRNA-26a targets ten eleven translocation enzymes and is regulated during pancreatic cell differentiation. Proc Natl Acad Sci USA 2013;110:17892-7.

* [31] Morán I, Akerman İ, van de Bunt M, et al. Human $\beta$ Cell Transcriptome Analysis Uncovers lncRNAs That Are Tissue-Specific, Dynamically Regulated, and Abnormally Expressed in Type 2 Diabetes. Cell Metab 2012;16:435-48.

[32] Ku GM, Kim H, Vaughn IW, et al. Research resource: RNA-Seq reveals unique features of the pancreatic $\beta$-cell transcriptome. Mol Endocrinol 2012;26:1783-92.

[33] Martínez-Romero C, Rooman I, Skoudy A, et al. The epigenetic regulators Bmi1 and Ring1B are differentially regulated in pancreatitis and pancreatic ductal adenocarcinoma. J Pathol 2009;219:205-13.

[34] Clair JM-S, Soydaner-Azeloglu R, Lee KE, et al. EZH2 couples pancreatic regeneration to neoplastic progression. Genes Dev 2012;26:439-44.

* [35] Bramswig NC, Everett LJ, Schug J, et al. Epigenomic plasticity enables human pancreatic $\alpha$ to $\beta$ cell reprogramming. J Clin Invest 2013;123:1275-84.

[36] Dhawan S, Georgia S, Tschen S, et al. Pancreatic beta cell identity is maintained by DNA methylationmediated repression of Arx. Dev Cell 2011;20:419-29.

[37] Mosley AL, Ozcan S. Glucose regulates insulin gene transcription by hyperacetylation of histone h4. J Biol Chem 2003;278:19660-6.

[38] Wang H-W, Breslin MB, Lan MS. Pdx-1 modulates histone H4 acetylation and insulin gene expression in terminally differentiated alpha-TC-1 cells. Pancreas 2007;34:248-53.

[39] Mosley AL, Ozcan S. The pancreatic duodenal homeobox-1 protein (Pdx-1) interacts with histone deacetylases Hdac-1 and Hdac-2 on low levels of glucose. J Biol Chem 2004;279:54241-7.

[40] Deering TG, Ogihara T, Trace AP, et al. Methyltransferase Set7/9 Maintains Transcription and Euchromatin Structure at Islet-Enriched Genes. Diabetes 2009;58:185-93.

[41] Evans-Molina C, Robbins RD, Kono T, et al. Peroxisome proliferator-activated receptor gamma activation restores islet function in diabetic mice through reduction of endoplasmic reticulum stress and maintenance of euchromatin structure. Mol Cell Biol 2009;29:2053-67.

[42] Singer RA, Arnes L, Sussel L. Noncoding RNAs in $\beta$ cell biology. Curr Opin Endocrinol Diabetes Obes 2015;22:77-85.

[43] Kalis M, Bolmeson C, Esguerra JLS, et al. Beta-Cell Specific Deletion of Dicer1 Leads to Defective Insulin Secretion and Diabetes Mellitus. PLoS ONE 2011;6:e29166.

[44] Klein D, Misawa R, Bravo-Egana V, et al. MicroRNA expression in alpha and beta cells of human pancreatic islets. PLoS ONE 2013;8:e55064.

[45] Poy MN, Hausser J, Trajkovski M, et al. miR-375 maintains normal pancreatic alpha- and beta-cell mass. Proc Natl Acad Sci USA 2009;106:5813-8.

[46] Melkman-Zehavi T, Oren R, Kredo-Russo S, et al. miRNAs control insulin content in pancreatic $\beta$-cells via downregulation of transcriptional repressors. EMBO J 2011;30:835-45.

[47] Pullen TJ, Rutter GA. Roles of IncRNAs in pancreatic beta cell identity and diabetes susceptibility. Front Genet 2014;5:193.

[48] Karnik SK, Chen H, McLean GW, et al. Menin controls growth of pancreatic beta-cells in pregnant mice and promotes gestational diabetes mellitus. Science 2007;318:806-9.

[49] Chen H, Gu X, Su I -hsi., et al. Polycomb protein Ezh2 regulates pancreatic beta-cell Ink4a/Arf expression and regeneration in diabetes mellitus. Genes Dev 2009;23:975-85.

[50] Dhawan S, Tschen S-I, Bhushan A. Bmi-1 regulates the Ink4a/Arf locus to control pancreatic $\beta$-cell proliferation. Genes Dev 2009;23:906-11.

* [51] Zhou JX, Dhawan S, Fu H, et al. Combined modulation of polycomb and trithorax genes rejuvenates $\beta$ cell replication. J Clin Invest 2013;123:4849-58.

[52] Schiesser JV, Wells JM. Generation of $\beta$ cells from human pluripotent stem cells: are we there yet? Ann N Y Acad Sci 2014;1311:124-37.

[53] Pagliuca FW, Millman JR, Gürtler M, et al. Generation of Functional Human Pancreatic $\beta$ Cells In Vitro. Cell 2014;159:428-39.

[54] Rezania A, Bruin JE, Arora P, et al. Reversal of diabetes with insulin-producing cells derived in vitro from human pluripotent stem cells. Nat Biotech 2014;32:1121-33.

[55] Xie R, Everett LJ, Lim H-W, et al. Dynamic chromatin remodeling mediated by polycomb proteins orchestrates pancreatic differentiation of human embryonic stem cells. Cell Stem Cell 2013;12:224-37.

[56] Zentner GE, Tesar PJ, Scacheri PC. Epigenetic signatures distinguish multiple classes of enhancers with distinct cellular functions. Genome Res 2011;21:1273-83.

[57] Ong C-T, Corces VG. Enhancers: emerging roles in cell fate specification. EMBO Rep 2012;13:423-30.

[58] Papp B, Plath K. Epigenetics of Reprogramming to Induced Pluripotency. Cell 2013;152:1324-43. 
[59] Wang A, Yue F, Li Y, et al. Epigenetic Priming of Enhancers Predicts Developmental Competence of hESC-Derived Endodermal Lineage Intermediates. Cell Stem Cell 2015;16:386-99.

* [60] Pennarossa G, Maffei S, Campagnol M, et al. Brief demethylation step allows the conversion of adult human skin fibroblasts into insulin-secreting cells. Proc Natl Acad Sci USA 2013;110:8948-53.

[61] Katz LS, Geras-Raaka E, Gershengorn MC. Reprogramming adult human dermal fibroblasts to isletlike cells by epigenetic modification coupled to transcription factor modulation. Stem Cells Dev 2013;22:2551-60.

[62] Johannesson B, Sagi I, Gore A, et al. Comparable frequencies of coding mutations and loss of imprinting in human pluripotent cells derived by nuclear transfer and defined factors. Cell Stem Cell 2014;15:634-42.

* [63] DIAbetes Genetics Replication And Meta-analysis (DIAGRAM) Consortium, Asian Genetic Epidemiology Network Type 2 Diabetes (AGEN-T2D) Consortium, South Asian Type 2 Diabetes (SAT2D) Consortium, et al. Genome-wide trans-ancestry meta-analysis provides insight into the genetic architecture of type 2 diabetes susceptibility. Nat Genet 2014;46:234-44.

[64] Park JH, Stoffers DA, Nicholls RD, Simmons RA. Development of type 2 diabetes following intrauterine growth retardation in rats is associated with progressive epigenetic silencing of Pdx1. J Clin Invest 2008;118:2316-24.

[65] Sandovici I, Smith NH, Nitert MD, et al. Maternal diet and aging alter the epigenetic control of a promoter-enhancer interaction at the $\mathrm{Hnf} 4 \mathrm{a}$ gene in rat pancreatic islets. Proc Natl Acad Sci USA 2011;108:5449-54.

[66] Prokopenko I, Poon W, Mägi R, et al. A central role for GRB10 in regulation of islet function in man. PLoS Genet 2014;10:e1004235.

[67] Yang BT, Dayeh TA, Volkov PA, et al. Increased DNA methylation and decreased expression of PDX1 in pancreatic islets from patients with type 2 diabetes. Mol Endocrinol 2012;26:1203-12.

[68] Ling C, Del Guerra S, Lupi R, et al. Epigenetic regulation of PPARGC1A in human type 2 diabetic islets and effect on insulin secretion. Diabetologia 2008;51:615-22.

[69] Volkmar M, Dedeurwaerder S, Cunha DA, et al. DNA methylation profiling identifies epigenetic dysregulation in pancreatic islets from type 2 diabetic patients. EMBO J 2012;31:1405-26.

* [70] Dayeh T, Volkov P, Salö S, et al. Genome-Wide DNA Methylation Analysis of Human Pancreatic Islets from Type 2 Diabetic and Non-Diabetic Donors Identifies Candidate Genes That Influence Insulin Secretion. PLoS Genet 2014;10.

[71] Dayeh TA, Olsson AH, Volkov P, et al. Identification of CpG-SNPs associated with type 2 diabetes and differential DNA methylation in human pancreatic islets. Diabetologia 2013;56:1036-46.

[72] Pasquali L, Gaulton KJ, Rodríguez-Seguí SA, et al. Pancreatic islet enhancer clusters enriched in type 2 diabetes risk-associated variants. Nat Genet 2014;46:136-43.

[73] Bhandare R, Schug J, Le Lay J, et al. Genome-wide analysis of histone modifications in human pancreatic islets. Genome Res 2010;20:428-33.

[74] Stitzel ML, Sethupathy P, Pearson DS, et al. Global epigenomic analysis of primary human pancreatic islets provides insights into type 2 diabetes susceptibility loci. Cell Metab 2010;12:443-55.

[75] Van Arensbergen J, García-Hurtado J, Moran I, et al. Derepression of Polycomb targets during pancreatic organogenesis allows insulin-producing beta-cells to adopt a neural gene activity program. Genome Res 2010;20:722-32.

* [76] Kameswaran V, Bramswig NC, McKenna LB, et al. Epigenetic regulation of the DLK1-MEG3 microRNA cluster in human type 2 diabetic islets. Cell Metab 2014;19:135-45.

[77] Latreille M, Hausser J, Stützer I, et al. MicroRNA-7a regulates pancreatic $\beta$ cell function. J Clin Invest 2014;124:2722-35.

[78] Zhao X, Mohan R, Özcan S, Tang X. MicroRNA-30d induces insulin transcription factor MafA and insulin production by targeting mitogen-activated protein 4 kinase 4 (MAP4K4) in pancreatic $\beta$-cells. J Biol Chem 2012;287:31155-64.

[79] Bao L, Fu X, Si M, et al. MicroRNA-185 targets SOCS3 to inhibit beta-cell dysfunction in diabetes. PLoS ONE 2015;10:e0116067.

[80] Erener S, Mojibian M, Fox JK, et al. Circulating miR-375 as a biomarker of $\beta$-cell death and diabetes in mice. Endocrinology 2013;154:603-8.

[81] Higuchi C, Nakatsuka A, Eguchi J, et al. Identification of circulating miR-101, miR-375 and miR-802 as biomarkers for type 2 diabetes. Metab Clin Exp 2015;64:489-97.

[82] Kameswaran V, Kaestner KH. The Missing lnc(RNA) between the pancreatic $\beta$-cell and diabetes. Front Genet 2014;5:200.

[83] Guerra C, Barbacid M. Genetically engineered mouse models of pancreatic adenocarcinoma. Mol Oncol 2013;7:232-47.

[84] Guo M, Jia Y, Yu Z, et al. Epigenetic changes associated with neoplasms of the exocrine and endocrine 
pancreas. Discov Med 2014;17:67-73.

[85] Nones K, Waddell N, Song S, et al. Genome-wide DNA methylation patterns in pancreatic ductal adenocarcinoma reveal epigenetic deregulation of SLIT-ROBO, ITGA2 and MET signaling. Int J Cancer 2014;135:1110-8.

[86] Vincent A, Hong S-M, Hu C, et al. Epigenetic silencing of EYA2 in pancreatic adenocarcinomas promotes tumor growth. Oncotarget 2014;5:2575-87.

[87] Dutruel C, Bergmann F, Rooman I, et al. Early epigenetic downregulation of WNK2 kinase during pancreatic ductal adenocarcinoma development. Oncogene 2014;33:3401-10.

[88] Gao W, Gu Y, Li Z, et al. miR-615-5p is epigenetically inactivated and functions as a tumor suppressor in pancreatic ductal adenocarcinoma. Oncogene 2015;34:1629-40.

[89] Jiao Y, Shi C, Edil BH, et al. DAXX/ATRX, MEN1 and mTOR Pathway Genes are Frequently Altered in Pancreatic Neuroendocrine Tumors. Science 2011;331:1199-203.

[90] Wang W, Gao J, Man X-H, et al. Significance of DNA methyltransferase-1 and histone deacetylase-1 in pancreatic cancer. Oncol Rep 2009;21:1439-47.

[91] Grandinetti KB, Jelinic P, DiMauro T, et al. Sin3B expression is required for cellular senescence and is up-regulated upon oncogenic stress. Cancer Res 2009;69:6430-7.

[92] Rielland M, Cantor DJ, Graveline R, et al. Senescence-associated SIN3B promotes inflammation and pancreatic cancer progression. J Clin Invest 2014;124:2125-35.

[93] DiMauro T, Cantor DJ, Bainor AJ, David G. Transcriptional repression of Sin3B by Bmi-1 prevents cellular senescence and is relieved by oncogene activation. Oncogene, 2015;34:4011-7

[94] $\mathrm{Xu} \mathrm{J,} \mathrm{Zhu} \mathrm{W,} \mathrm{Xu} \mathrm{W,} \mathrm{et} \mathrm{al.} \mathrm{Up-regulation} \mathrm{of} \mathrm{MBD1} \mathrm{promotes} \mathrm{pancreatic} \mathrm{cancer} \mathrm{cell} \mathrm{epithelial-}$ mesenchymal transition and invasion by epigenetic down-regulation of E-cadherin. Curr Mol Med 2013;13:387-400.

[95] Chen S, Chen J, Zhan Q, et al. H2AK119Ub1 and H3K27Me3 in molecular staging for survival prediction of patients with pancreatic ductal adenocarcinoma. Oncotarget 2014;5:10421-33.

[96] Jia J, Parikh H, Xiao W, et al. An integrated transcriptome and epigenome analysis identifies a novel candidate gene for pancreatic cancer. BMC Med Genomics 2013;6:33.

[97] Park JY, Helm J, Coppola D, et al. MicroRNAs in pancreatic ductal adenocarcinoma. World J Gastroenterol 2011;17:817-27.

[98] Caponi S, Funel N, Frampton AE, et al. The good, the bad and the ugly: a tale of miR-101, miR-21 and miR-155 in pancreatic intraductal papillary mucinous neoplasms. Ann Oncol 2013;24:734-41.

[99] Nakahara O, Takamori H, Iwatsuki M, et al. Carcinogenesis of intraductal papillary mucinous neoplasm of the pancreas: loss of microRNA-101 promotes overexpression of histone methyltransferase EZH2. Ann Surg Oncol 2012;19 Suppl 3:S565-71. 